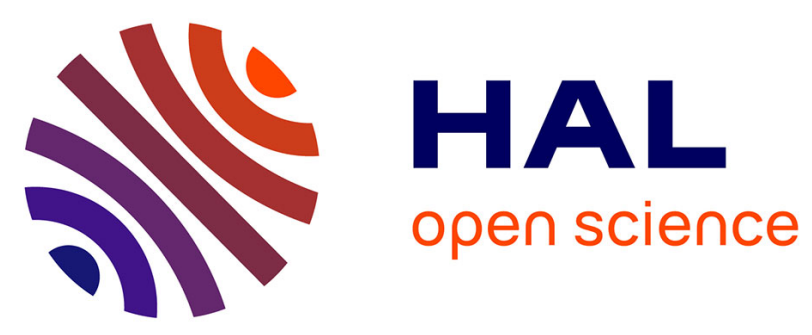

\title{
The symptom of low mood in the prodromal stage of mild cognitive impairment and dementia: a cohort study of community-dwelling elderly
}

Barbara Caracciolo, Lars Bäckman, Roberto Monastero, B. Winblad, Laura Fratiglioni

\section{To cite this version:}

Barbara Caracciolo, Lars Bäckman, Roberto Monastero, B. Winblad, Laura Fratiglioni. The symptom of low mood in the prodromal stage of mild cognitive impairment and dementia: a cohort study of community-dwelling elderly. Journal of Neurology, Neurosurgery and Psychiatry, 2011, 82 (7), pp.788. 10.1136/jnnp.2010.225003. hal-00606281

\section{HAL Id: hal-00606281 \\ https://hal.science/hal-00606281}

Submitted on 6 Jul 2011

HAL is a multi-disciplinary open access archive for the deposit and dissemination of scientific research documents, whether they are published or not. The documents may come from teaching and research institutions in France or abroad, or from public or private research centers.
L'archive ouverte pluridisciplinaire HAL, est destinée au dépôt et à la diffusion de documents scientifiques de niveau recherche, publiés ou non, émanant des établissements d'enseignement et de recherche français ou étrangers, des laboratoires publics ou privés. 


\section{The symptom of low mood in the prodromal stage of mild cognitive} impairment and dementia: a cohort study of community-dwelling elderly

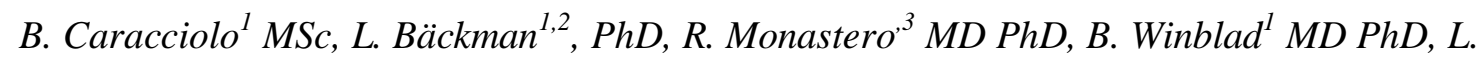
Fratiglioni $^{1,2} M D P h D$

\section{Authors' affiliations:}

1. Aging Research Center, Department of Neurobiology, Care Sciences and Society, Karolinska Institutet and Stockholm University, Stockholm, Sweden; 2. Stockholm

Gerontology Research Center, Stockholm, Sweden; 3. Department of Clinical Neuroscience, Section of Neurology, University of Palermo, Palermo, Italy

\section{Correspondence to:}

Barbara Caracciolo, MSc

Aging Research Center, Karolinska Institutet, Gävlegatan 16, 11330 Stockholm, Sweden Phone: +46 8 6905827; Fax: +4686906889; E-mail: barbara.caracciolo@ki.se

Word count: 3629 words.

Search terms: dementia; depression; MCI (mild cognitive impairment); population-based; early symptoms. 


\begin{abstract}
Objective: To investigate the symptom of low mood as a predictor of mild cognitive impairment (MCI) and its progression to dementia, taking into account: i) MCI severity, ii) time of assessment, iii) interaction with other factors.

Methods: 764 cognitively healthy elderly living in the community, from the Kungsholmen Project. Participants were assessed by direct interview to detect low mood. Subjects were then followed for six years to identify those who developed MCI. People with incident MCI were followed for a further three years to assess progression to dementia.
\end{abstract}

Results: People with low mood at baseline had a 2.7-fold (95\% Confidence Interval [CI] 1.93.7) increased risk of developing MCI at follow-up. The association was stronger for amnestic MCI (aMCI: Hazard Ratio [HR] 5.8; 95\% CI 3.1-10.9) compared to global cognitive impairment (other cognitive impairment no dementia, oCIND: HR 2.2; 95\% CI 1.5-3.3). ApoE- $\varepsilon 4$ interacted with low mood in a synergistic fashion, increasing the risk of aMCI, while no interaction with psychiatric, vascular, frailty-related, and psychosocial factors was observed. Low mood at baseline, as opposed to low mood co-occurring with MCI, was associated with a 5.3-fold (95\% CI 1.2-23.3) increased risk of progression to dementia in aMCI. In contrast, no association was found in oCIND.

Conclusion: Low mood was more strongly associated with aMCI than with global cognitive impairment. Progression toward dementia was predicted only by low mood manifest in the prodromal stage of MCI. These findings indicate that low mood is particularly prominent in the very early stages of cognitive decline. 


\section{INTRODUCTION}

Depression is the most studied neuropsychiatric feature of mild cognitive impairment and its association with cognitive deterioration has been shown both cross-sectionally and longitudinally. ${ }^{1}$ Prevalence of depression is higher in MCI and dementia than in cognitively healthy subjects. ${ }^{2}$ Subclinical syndromes of depression and isolated depressive symptoms are more common than clinical depression in both MCI and dementia. Interestingly, the most prevalent symptom of depression in MCI is low mood, ${ }^{1}$ whereas in dementia apathy is predominant. ${ }^{1}$ Longitudinally, depressive symptoms in cognitively healthy persons have been consistently associated with the development of MCI. ${ }^{3-7}$ In contrast, studies on the progression of MCI toward dementia have produced conflicting evidence. Out of ten studies, ${ }^{4,8-16}$ three found that depressive symptoms increased the risk of progressing to dementia; ${ }^{4,14,16}$ two reported an association only in women or for isolated symptoms; ${ }^{12,13}$ three found no association; ${ }^{10-11,15}$ and two recent studies reported that the presence of depressive symptoms decreased the risk of developing dementia. ${ }^{8-9}$

The discrepancy between studies on MCI development and studies on MCI progression may have alternative explanations. First, depressive symptoms may have a different role in different stages of the cognitive decline process. Specifically, symptoms of depression that are the expression of underlying neurodegenerative pathology ${ }^{17}$ may be prominent during the transition between healthy cognition and initial cognitive impairment, but lose their importance in more advanced stages of cognitive decline. Thus, depressive symptoms may be differentially related to MCI types of different severity that represent different stages of the dementing process. In amnestic MCI (aMCI), ${ }^{18-19}$ for instance, memory deficits are present in the background of otherwise preserved global cognitive functioning, whereas in other definitional categories, such as other cognitive impairment no dementia (oCIND), ${ }^{20-21}$ the cognitive functioning is globally compromised, although not yet to a degree sufficient to 
fulfill the criteria for a diagnosis of dementia. Therefore, oCIND can be interpreted as a more advanced stage of cognitive impairment compared to aMCI.

Second, most studies investigating the progression of MCI to dementia have focused on depressive symptoms co-occurring with MCI. Indeed, if low mood is an early feature of dementia-related neuropathology, its onset should precede the stages when cognitive impairment becomes manifest, first as MCI and later as dementia. When MCI or dementia is detectable, depressive symptoms could also result from a subjective reaction to the cognitive problems, leading to reverse causality. Therefore, as depressive symptoms may be due to different mechanisms, they may have different odds of progressing toward dementia in people with MCI. Heterogeneity of depressive symptoms might also result in different responses to antidepressant drugs. It has been shown that depressive symptoms in MCI are particularly resistant to treatment. ${ }^{16}$ For these reasons, it is important to pay special attention to the time of assessment of the depressive symptoms when studying the association with MCI and the impact on progression from MCI to dementia.

Third, depression as a prodromal feature of cognitive impairment might have characteristics that do not coincide with the typical depressive syndrome, as described by depression interviews, scales and questionnaires. Indeed, isolated depressive symptoms are common in MCI and low mood is the most prevalent symptom. ${ }^{1}$ Moreover, in a recent clinically-based study, low mood predicted progression of MCI to AD better than scores from a depression scale. $^{13}$

Finally, to understand the nature of the relationship between depressive symptoms and MCI, it is important to unfold the pattern of interactions with other factors (e.g., genetic, psychiatric vascular, frailty-related and psychosocial) that are relevant for both depression and MCI and that might modify their association. 
The present paper aimed at investigating the symptom of low mood as a predictor of MCI and its progression to dementia in the framework of a large community-based study of the elderly, taking into account: i) MCI severity, ii) time of assessment, iii) interaction with other factors.

\section{METHODS}

\section{STUDY POPULATION}

Data were gathered from the Kungsholmen Project, a community-based, prospective study of persons aged 75 years and above, who lived in Kungsholmen, Stockholm, Sweden, at the end of $1987 .{ }^{22}$ As illustrated in Figure 1, out of 1700 participants at baseline (1987-1989) 40 persons were excluded due to very low global cognitive status or unknown educational background. To obtain a cognitively healthy cohort, we further excluded: a) prevalent dementia cases $(n=225)$; and $b)$ all prevalent MCI cases ( $n=337)$. Of the remaining 1098 persons, $199(18 \%)$ died, and $135(12 \%)$ moved or discontinued their participation before the first follow-up examination. The resulting 764 cognitively healthy cohort members were followed for six years, during which two clinical examinations took place with an interval of three years (first follow-up: 1994-1996; second follow-up: 1997-1998). To ascertain progression toward dementia, incident MCI cases were followed for three further years (third follow-up:1999-2000).

Between the first and the second follow-up examinations, 28 (30\%) cases died and six (6\%) refused or moved. Between the second and third follow-up examinations 23 (35\%) cases died and four $(6 \%)$ refused or moved.

All phases of the Kungsholmen Project have been approved by the Ethical Committee of Karolinska Institutet, and informed consent was obtained from all participants. 


\section{DATA COLLECTION}

All study participants underwent a comprehensive clinical, medical, cognitive, and psychosocial examination both at baseline and at follow-ups, as described in detail elsewhere. ${ }^{20,22}$ Information on medical history was taken from the inpatient register system, which encompasses all hospitals in Stockholm from 1969 onward. The International Classification of Disease, 8 th revision (ICD-8) was used in the register system until $1986 ;{ }^{23}$ since 1987 the ICD-9th revision (ICD-9) was employed. ${ }^{24}$ Information on drug use (in the preceding two weeks) and blood samples were collected at each examination.

\section{LOW MOOD SYMPTOM}

We defined the symptom of low mood as a report of perceived sadness. At baseline, nurses assessed mental health as part of a general health status interview consisting of questions with yes/no answers. The questionnaire investigated different health-related symptoms and included items on depressive symptoms, such as low mood, anxiety, feelings of loneliness, sleeping disturbances, reduced appetite, and tiredness. All of these symptoms except loss of appetite loaded on the same factor, as shown by a factor analysis with varimax rotation performed at baseline. Low mood had the highest loadings on this depression factor (0.81), followed by feeling lonely (0.70) and anxiety (0.64).

At follow-ups, all patients underwent a structured psychiatric interview performed by physicians and based on the Comprehensive Psychopathological Rating Scale (CPRS). ${ }^{25}$ Report of perceived sadness was drawn from the CPRS interview, and was graded in degree of severity from a scale from 0 (no perceived sadness) to 6 (extreme sadness). ${ }^{26}$ The item was used both as an ordinal variable and as a categorical variable. In the latter case, the item was dichotomized into low mood, for all scores exceeding 0 , versus no low mood, for a score equal to 0 . 


\section{DEFINITION OF MCI}

People with MCI included all nondemented participants who fulfilled amnestic MCI $(\mathrm{aMCI})^{18-19}$ or other cognitive impairment no dementia (oCIND) $)^{20-21}$ criteria. aMCI was defined according to standard criteria ${ }^{18-19}$ and operationalized according to previous research ${ }^{20}$ as follows: 1) Memory complaints were assessed based on memory problems reported by the subjects or informants; 2) Normal general cognitive function was defined as scoring 1 SD above the age- and education-adjusted Mini Mental State Examination mean (MMSE); ${ }^{27} 3$ ) Absence of dementia was verified by clinical examination; 4) Normal activities of daily living was characterised by no impairment in Katz' Activities of Daily Living scale; ${ }^{28}$ and 5) Objective memory impairment was defined as scoring 1.5 SD below the age- and educationadjusted mean in a verbal memory task (free recall of slowly and rapidly presented words). ${ }^{29}$ All cases with global cognitive impairment that did not fulfil criteria for dementia were classified as oCIND ${ }^{21}$ and operationalized according to previous research ${ }^{20}$ as follows: 1) Impaired general cognitive function, defined as scoring $1 \mathrm{SD}$ or more below age- and education-adjusted means on the MMSE derived from the dementia-free population at baseline; and 2) Absence of dementia was verified by clinical examination. aMCI and oCIND were mutually exclusive in the present study.

\section{DIAGNOSIS OF DEMENTIA}

Dementia was diagnosed according to the Diagnostic and Statistical Manual of Mental Disorders, Revised Third Edition (DSM-III-R) criteria, ${ }^{30}$ following a three-step procedure, as described in detail elsewhere. ${ }^{22}$ The dementia diagnoses for dead subjects were made by physicians consulting clinical records and death certificates. ${ }^{31}$

In the present study, a broad category including all dementia cases, regardless of dementia type was considered. However, Alzheimer's disease (AD) was the most common form of dementia detected, accounting for nearly $80 \%$ of all cases. 


\section{COVARIATES}

Medical conditions derived from the inpatient register database were: history of psychosis (ICD-8 and ICD-9 codes 291-298), which included episodic mood disorders; history of cerebrovascular disease (ICD-8 and ICD-9 codes 430-438); history of heart disease (ICD-8 or ICD-9 codes 410-414 and 427-428); hip fracture (ICD-8 and ICD-9 codes 820, 821), and diabetes (ICD-8 and ICD-9 code 250). Data on diabetes were integrated with information from other sources, as descried elsewhere (32). High blood pressure was defined as $\geq 180$ arterial blood pressure (i.e., systolic Korotkoff phase I and diastolic phase V). Multimorbidity was defined as having two or more chronic conditions. Polypharmacy was defined as the use of more than 5 drugs. ${ }^{33}$ Psychotropic drugs use was defined as using one versus none of the following psychoactive drugs: neuroleptics (ATC code: N05A), anxiolytics (ATC code: N05B), other tranquilizers, hypnotics (ATC code: N05C), and antidepressants (ATC code: N06A). APOE genotyping was carried out using a standard polymerase chain reaction (34) and dichotomized as having at least one ApoE- $\varepsilon 4$ allele versus having none. Social network was coded according to previous research as a four-level ordinal variable (extensive/moderate/limited/poor social network). ${ }^{35}$

\section{DATA ANALYSIS}

INCIDENCE OF MCI AMONG COGNITIVELY HEALTHY PEOPLE. The cognitively healthy cohort was followed for six years to detect new cases of MCI. Subjects were considered at risk of MCI until they i) were classified for the first time as MCI or demented; ii) dropped-out of the study due to refusal or moving; or iii) died.

Differences in subject characteristics were tested with Pearson's Chi Square. Independent Cox models were used to estimate the Hazard ratio (HR) and 95\% confidence intervals (CI) of 
developing MCI in relation to baseline low mood (baseline LM). For cases of MCI detected at the first wave of examinations, the baseline for low mood exposure was set at the study baseline. For cases of MCI detected at second wave of examinations, the baseline for low mood exposure was set at the second wave (first follow-up). The relative risks of MCI in relation to baseline low mood were comparable between the two waves, therefore Cox regression analysis was performed using data from both waves. In order to preserve the threeyear follow-up exposure for MCI, baseline LM and all other variables varying with time were entered as time-dependent variables. Low mood co-occurring with incident MCI (follow-up LM) was used as a covariate. Multiplicative interactions were tested within Cox regression. To test for additive interactions, the attributable proportion (AP) due to interaction was calculated together with the $95 \% \mathrm{CI}^{36}$

INCIDENCE OF DEMENTIA AMONG COGNITIVELY HEALTHY PEOPLE. Dementia developed in cognitively healthy people not previously classified as MCI was used as a competitive outcome and the relation with low mood was analyzed with the same methodology described above.

EVOLUTION OF MCI. Persons who developed MCI were followed for three further years to assess their progression toward dementia. Independent Cox regression models were run to evaluate the HR and $95 \%$ CI of dementia in subjects with MCI in relation to baseline and follow-up low mood. Adjustment and interactions with other covariates were tested as described above. 
Missing values were imputed with Multiple Imputation based on available information on other covariates, ${ }^{37}$ using Stata 9.0. All other analyses were performed using SPSS, with an alpha level of $\mathrm{p}<0.05$.

\section{RESULTS}

INCIDENCE OF MCI AMONG COGNITIVELY HEALTHY PEOPLE. During the 3711.5 person-years (minimum 1.2; maximum 8.2) of follow-up, 160 persons developed MCI. Of these, 40 were classified as aMCI and 120 as oCIND. Characteristics of the cohort and the cases are shown in Table 1. Fifty-three percent $(n=21)$ of aMCI and 31 percent $(n=37)$ of oCIND occurred in people with baseline LM. When considering aMCI and oCIND together, the incidence of MCI in people with baseline LM was about 2.5 times higher than that detected among people without baseline LM, and this ratio was constant across the two waves of examinations (Figure 2). Cox regression analysis performed using data from both waves confirmed the increased risk of all outcomes in relation to the presence of baseline LM, although the association was stronger for aMCI. Adjustment for socioeconomic characteristics and other covariates, including follow-up LM, history of psychosis, psychotropic drug use, ApoE- $\varepsilon 4$ allele, history of cerebrovascular disease, heart disease, diabetes, high blood pressure, hip fracture, multimorbidity, and polypharmacy, did not substantially change the results and HRs remained stable even after multi-adjustment (Table 2).

Stratified analyses showed that the increased risk of aMCI and oCIND associated with baseline LM was not substantially modified by follow-up LM nor by the other factors (as listed above). However, an additive interaction was found between baseline LM and ApoE- $\varepsilon 4$ allele for the risk of developing aMCI (Attributable Proportion: 0.5, 95\% CI: 0.1-0.96) (Figure 3), but not for the risk of developing oCIND. No other interactions were observed. 
INCIDENCE OF DEMENTIA AMONG COGNITIVELY HEALTHY PEOPLE. During the follow-up, 158 cognitively healthy persons developed dementia by-passing MCI. In these people, the HR for low mood was 1.6 (95\% CI 1.1-2.3) after adjustment for age, sex, and, education. Further adjustment for the other covariates did not affect the relationship with baseline LM. However, the association was no longer significant after adjustment for followup LM (HR 1.4, 95\% CI 0.9-2.0) and in the multi-adjusted model (HR 1.3, 95\% CI 0.9-1.9).

EVOLUTION OF MCI. During the 1354.8 person-years (minimum 0.7; maximum 4.5) of follow-up, 50 people with MCI progressed to dementia. Eleven cases of dementia occurred in people with aMCI and 39 cases of dementia occurred in people with oCIND. Among the aMCI persons who progressed to dementia, eight (70\%) had baseline LM, detected before the development of aMCI, and three (27\%) had follow-up LM, which was detected at the time of aMCI classification. Among the oCIND that progressed to dementia, 14 (33\%) had baseline LM and six (15\%) had follow-up LM. Cox regression analysis confirmed the association of baseline LM with an increased risk of progression to dementia in people with aMCI (Table 3). The association was strengthened after further adjustment for ApoE- $\varepsilon 4$ allele. Adjustment for other factors did not substantially change the association. On the other hand, follow-up LM was not associated with an increased risk of progression to dementia in people with aMCI (Table 3). Progression to dementia in people with oCIND was linked neither to baseline nor to follow-up LM (Table 3).

\section{DISCUSSION}

In our large, prospective, community-based study, low mood reported three years before MCI detection substantially increased the risk of developing MCI. In particular, low mood was associated with a 5.8-fold increased risk of aMCI, a 2.2-fold increased risk of oCIND, and a 
2.7-fold increased risk of All-MCI, after adjustment for sociodemographic factors. These findings are in line with previous evidence from other longitudinal studies, which found an association between baseline depressive symptoms and incident MCI, with estimates ranging from a 1.2 to a 16 -fold increased risk of $\mathrm{MCI}^{3-7}$

The excess risk of aMCI associated with low mood was almost triple that for oCIND (HR 5.8 versus 2.2), suggesting the presence of a gradient in the relationship between low mood and cognitive impairment severity. Indeed, in our study the definition of amnestic MCI excluded people with global cognitive impairment (MMSE mean=26). On the other hand, oCIND included more severely impaired cases with global cognitive impairment (MMSE mean=21), who did not fulfill criteria for dementia. In addition, we also observed a group of people that rapidly progressed to dementia by-passing $\mathrm{MCI}(\mathrm{MMSE}$ mean=8), for whom the excess risk associated with low mood was $60 \%$, lower than that observed in both aMCI and oCIND. Interestingly, we found a synergistic action of low mood and ApoE- $\varepsilon 4$, with increased risk of aMCI in people with both baseline low mood and at least one ApoE- $\varepsilon 4$ allele. This is in line with results from a study on primary care patients ${ }^{5}$ and supports the hypothesis that low mood may be related to AD-type neuropathology. ${ }^{17,38}$ Conversely, none of the other factors considered in our investigation interacted with low mood. In particular, no modification of the relationship of baseline low mood with MCI was observed by taking into account history of psychosis, psychotropic drug use, and vascular factors. Indeed, low mood in prodromal MCI can be independent from history of depression. ${ }^{5}$ It has also been observed that depressive symptoms in MCI are particularly resistant to treatment. ${ }^{16,39}$ On the other hand, the lack of interaction of low mood with vascular factors confirms previous findings from the Cardiovascular Health Study and does not support the "vascular depression hypothesis". 6 Low mood was associated with a 5.3-fold increased risk of progression to dementia in people with aMCI. This finding is in line with some progression studies of $\mathrm{MCI}^{4,12-14,16}$ and is at odds 
with others. ${ }^{8-11,15}$ Indeed, most of the studies that found no or inverse association focused on depressive symptoms measured at the same time of MCI detection. ${ }^{8,9,11,15}$ We showed that only low mood measured at baseline, three years before the detection of MCI, predicted subsequent progression of MCI to dementia. Conversely, low mood that co-occurred with MCI did not predict further progression to dementia. This results support the hypothesis that the symptom of low mood may be relevant in the prodromal stage of MCI, losing its importance in more advanced stages, when the cognitive deficits are already manifested. Our findings do not confirm previous evidence from a report based on the Religious Order Study, ${ }^{10}$ which found no increase in depressive symptoms in the prodromal stage of dementia. Indeed, the generalizability of those results has been questioned, as religious order people might have higher resilience against depressive symptoms. ${ }^{40}$

Our study has many strengths, including the community-based design; the longitudinal assessment of the relationship between low mood and cognitive impairment; the comparison of the impact of low mood on different types and degrees of severity of cognitive impairment; the comparison of different times of assessment of low mood in relation with the progression of MCI to dementia; and the thorough assessment of the interaction of low mood with other factors. However, there are some limitations. First, it is difficult to disentangle the directionality of the association between low mood and cognitive impairment even in the framework of a longitudinal study. Reverse causation is always possible. Indeed, people might have performed poorly in the cognitive tests because they were depressed at the time of assessment. This could have generated a spurious association with baseline low mood if those people with low mood at time of MCI detection were already depressed at baseline. However, we controlled for this possibility and found no evidence of a confounding between baseline low mood and follow-up low mood. Second, the assessment of low mood was performed in different settings between the first and the second wave of examinations. Nonetheless, the 
figures regarding the occurrence of $\mathrm{MCI}$ in relation to low mood at the two measuring occasions are very similar, pointing to a substantial stability of the construct of low mood across different measurements. Third, grading of severity of low mood was not available for all participants. However, available information showed that the symptom was mild in most (91\%) of the people with low mood who developed MCI. Fourth, the cross-sectional assessment of low mood did not provide any information on duration. Nonetheless, the majority of people with MCI who reported low mood at baseline were euthymic on the subsequent three-year follow-up examination. Fifth, we used dementia as an outcome rather than dementia type. This was done considering the relatively small sample of MCI persons who progressed to dementia. However, in our population nearly $80 \%$ of all diagnosis of dementia was classified as probable or possible AD. Future investigations should try to expand our findings using dementia type as an outcome. Finally, in the present cohort there was a group of people who developed dementia without previously being classified as MCI. It is likely that the association between low mood and cognitive decline would have been further strengthened by using a follow-up interval short enough to observe the transitional stage of MCI in all the subjects who developed dementia.

Our results showed that low mood is a predictor of MCI development and its progression towards dementia, and confirmed our working hypothesis that low mood may be more closely related to early, rather than advanced, stages of cognitive decline. There are different possible explanations to this finding. One explanation would be that low mood is a risk factor for cognitive impairment and particularly memory functioning, which would likely be mediated by the interplay between psychosocial stress and the activity of the adreno-cortico-axis, which imbalance has known effects on neurogenesis and hippocampal physiology. ${ }^{41}$ Another possible explanation would be that low mood and MCI share a common neuropathogenic substrate. In this case the narrow time frame that we observed for the relationship between 
low mood and cognitive decline would be explained by the stage of the underlying neurodegenerative process. In earlier stages, when cognitive deterioration is not yet manifest, low mood could be one behavioral sign of neurodegeneration, as in the concept of "amyloidassociated depression". ${ }^{17}$ In more advanced stages, when the neurodegeneration has become more pervasive, MCI could represent a later manifestation of the process leading to dementia. In conclusion, although further studies are needed to disentangle the relative contribution of possible underlying mechanisms, cognitively healthy elderly presenting with the symptom of low mood should be closely monitored as they are at increased risk of MCI and subsequent progression to dementia. 
Caption to Figure 1. Study population: identification of the cognitively healthy cohort at baseline with follow-up data.

Caption to Figure 2. Crude incidence rates (IR) per 1000 person-years and 95\% confidence intervals of all mild cognitive impairment (All-MCI) in subjects with and without low mood at baseline. Data are shown for the first and second wave of examinations separately.

Caption to Figure 3. Hazard ratios (HR) with $95 \%$ confidence intervals (95\% CI) of amnestic mild cognitive impairment (aMCI) in relation to ApoE- $\varepsilon 4$ and baseline low mood. Subjects with no low mood at baseline and without any $\varepsilon 4$ allele were used as reference. Data are adjusted for age, sex, and education. 
Table 1. Study population and incident cases of amnestic mild cognitive impairment (aMCI), other cognitive impairment no dementia (oCIND), and overall mild cognitive impairment (All-MCI) by age, gender, and education.

\begin{tabular}{cccc}
\hline $\begin{array}{c}\text { Study } \\
\text { population }\end{array}$ & \multicolumn{3}{c}{$\begin{array}{c}\text { Incident } \\
\text { cases }\end{array}$} \\
\cline { 2 - 5 } & aMCI & oCIND & All-MCI \\
& $\mathrm{n}=40$ & $\mathrm{n}=120$ & $\mathrm{n}=160$ \\
\hline
\end{tabular}

Subjects characteristics

Age, baseline

$75-79$

$80+$

Gender

Women

Men

\section{Education}

High

Low
367 (48)

$397(52)$

$19(48)$

$61(51)$

$80(50)$

$21(52)$

$59(49)$

$80(50)$

$576(75) \quad 29(73) \quad 92(77) \quad 121(76)$

$188(25) \quad 11(28) \quad 28(23) \quad 39(24)$

$403(53)$

$27(68) \quad 55(46)$

$82(51)$

$361(47)$

$13(32)$

$65(54)$

$78(49)$ 
Table 2. Hazard ratios (HRs) and 95 percent confidence intervals (95\% CI) of amnestic mild cognitive impairment (aMCI), other cognitive impairment no dementia (oCIND), and overall mild cognitive impairment (All-MCI) in relation to baseline low mood (three years before MCI detection).

\begin{tabular}{lccc}
\hline aMCI & oCIND & All-MCI \\
& $\mathrm{n}=40$ & $\mathrm{n}=120$ & $\mathrm{n}=160$ \\
& $\mathrm{HR}$ & $\mathrm{HR}$ & $\mathrm{HR}$ \\
& $(95 \% \mathrm{CI})$ & $(95 \% \mathrm{CI})$ & $(95 \% \mathrm{CI})$ \\
\hline Basic adjustment* & 5.8 & 2.2 & 2.7 \\
& $(3.1-10.9)$ & $(1.5-3.3)$ & $(1.9-3.7)$ \\
Basic adjustment + follow-up LM** & & & \\
& 5.5 & 2.2 & 2.6 \\
Multiadjustment*** & $(2.9-10.6)$ & $(1.5-3.3)$ & $(1.9-3.7)$ \\
& & & 2.6 \\
& 6.2 & 2.2 & $(1.8-3.7)$
\end{tabular}

*Adjusted for: age, sex, education.

**Adjusted for: age, sex, education, and low mood assessed at the same time of MCI detection (follow-up LM).

***Simultaneous adjustment for age, sex, education, history of psychosis, psychotropic drug use, ApoE- $\varepsilon 4$ allele, history of cerebrovascular disease, heart disease, diabetes, high blood pressure, hip fracture, multimorbidity, and polypharmacy. 
Table 3. Hazard ratios (HRs) and 95\% confidence intervals (95\% CI) for three-year progression of amnestic mild cognitive impairment (aMCI), other cognitive impairment no dementia (oCIND), and overall mild cognitive impairment (All-MCI) to dementia in relation to low mood three years before detection of cognitive impairment (baseline LM) and to low mood reported at the time of detection of cognitive impairment (follow-up LM).

\begin{tabular}{|c|c|c|c|}
\hline & & \multicolumn{2}{|c|}{$\begin{array}{l}\text { Dementia } \\
(\mathbf{n}=\mathbf{5 0})\end{array}$} \\
\hline & & $\begin{array}{c}\text { HR }(95 \% \mathrm{CI}) \\
\text { for baseline LM }\end{array}$ & $\begin{array}{c}\text { HR }(95 \% \mathrm{CI}) \\
\text { for follow-up LM }\end{array}$ \\
\hline \multirow{2}{*}{ aMCI } & Basic adjustment* & $5.3(1.2-23.3)$ & $1.2(0.3-5.2)$ \\
\hline & Basic + ApoE- $\varepsilon 4 * *$ & $5.9(1.4-25.0)$ & $1.1(0.5-2.0)$ \\
\hline \multirow{2}{*}{ oCIND } & Basic adjustment* & $1.1(0.5-2.1)$ & $1.0(0.5-2.1)$ \\
\hline & Basic + ApoE- $\varepsilon 4 * *$ & $1.0(0.5-2.1)$ & $1.1(0.5-2.3)$ \\
\hline \multirow{2}{*}{ All-MCI } & Basic adjustment* & $1.4(0.8-2.4)$ & $1.0(0.5-2.0)$ \\
\hline & Basic + ApoE- $\varepsilon 4 * *$ & $1.4(0.8-2.4)$ & $1.1(0.5-2.0)$ \\
\hline
\end{tabular}

*Adjusted for: age, sex, education.

**Adjusted for: age, sex, education, and ApoE- $\varepsilon 4$ allelic status. 
Acknowledgements: We thank all staff of the Kungsholmen Project for their collaboration in data collection and management.

\section{Competing interests: None.}

Copyright: The Corresponding Author has the right to grant on behalf of all authors and does grant on behalf of all authors, an exclusive license (or non-exclusive for government employees) on a worldwide basis to the BMJ Publishing Group Ltd and its Licensees to permit this article (if accepted) to be published in Journal of Neurology, Neurosurgery \& Psychiatry and any other BMJPGL products to exploit all subsidiary rights, as set out in our licence (http://group.bmj.com/products/journals/instructions-for-authors/licence-forms).

Funding: This study was supported by grants from the Swedish Council for Working Life and Social Research, Regional agreement on medical training and clinical research (ALF) between Stockholm County Council and Karolinska Institutet, and the Swedish Brain Power Initiative. Private funding from Stiftelsen Gamla Tjänarinnor and Gun and Bertil Stohnes Foundation has also been provided. 


\section{References}

1. Monastero R, Mangialasche F, Camarda C, et al. A systematic review of neuropsychiatric symptoms in mild cognitive impairment. $J$ Alzheimers Dis 2009;18:11-30.

2. Geda YE, Roberts RO, Knopman DS, et al. Prevalence of neuropsychiatric symptoms in mild cognitive impairment and normal cognitive aging: population-based study. Arch Gen Psychiatry 2008;65:1193-8.

3. Rosenberg PB, Mielke MM, Xue QL, et al. Depressive symptoms predict incident cognitive impairment in cognitive healthy older women. Am J Geriatr Psychiatry 2010;18:204-11.

4. Stepaniuk J, Ritchie LJ, Tuokko H. Neuropsychiatric impairments as predictors of mild cognitive impairment, dementia, and Alzheimer's disease. Am J Alzheimers Dis Other Demen 2008;23:326-33.

5. Geda YE, Knopman DS, Mrazek DA, et al. Depression, apolipoprotein E genotype, and the incidence of mild cognitive impairment: a prospective cohort study. Arch Neurol 2006;63:435-40.

6. Barnes DE, Alexopoulos GS, Lopez OL, et al. Depressive symptoms, vascular disease, and mild cognitive impairment: findings from the Cardiovascular Health Study. Arch Gen Psychiatry 2006;63:273-79.

7. Lopez OL, Jagust WJ, Dulberg C, et al. Risk factors for mild cognitive impairment in the Cardiovascular Health Study Cognition Study: part 2. Arch Neurol 2003;60:139499.

8. Ramakers IH, Visser PJ, Aalten P, et al. Affective symptoms as predictors of Alzheimer's disease in subjects with mild cognitive impairment: a 10-year follow-up study. Psychol Med Epub 2009 Nov 11.

9. Vicini CB, Conti M, Zanetti M, et al. Differential impact of apathy and depression in the development of dementia in mild cognitive impairment patients. Dement Geriatr Cogn Disord 2009;27:390-98.

10. Wilson RS, Arnold SE, Beck TL, et al. Change in depressive symptoms during the prodromal phase of Alzheimer disease. Arch Gen Psychiatry 2008;65:439-45.

11. Panza F, Capurso C, D'Introno A, et al. Impact of depressive symptoms on the rate of progression to dementia in patients affected by mild cognitive impairment. The Italian Longitudinal Study on Aging. Int J Geriatr Psychiatry 2008;23:726-34.

12. Artero S, Ancelin ML, Portet F, et al. Risk profiles for mild cognitive impairment and progression to dementia are gender specific. J Neurol Neurosurg Psychiatry 2008;79:979-84. 
13. Houde M, Bergman $\mathrm{H}$, Whitehead $\mathrm{V}$, et al. A predictive depression pattern in mild cognitive impairment. Int J Geriatr Psychiatry 2008;23:1028-33.

14. Gabryelewicz T, Styczynska M, Luczywek E, et al. The rate of conversion of mild cognitive impairment to dementia: predictive role of depression. Int J Geriatr Psychiatry 2007;22:563-67.

15. Palmer K, Berger AK, Monastero R, et al. Predictors of progression from mild cognitive impairment to Alzheimer disease. Neurology 2007;68:1596-602.

16. Modrego PJ, Ferrandez J. Depression in patients with mild cognitive impairment increases the risk of developing dementia of Alzheimer type: a prospective cohort study. Arch Neurol 2004;61:1290-93.

17. Sun X, Steffens DC, Au R, et al. Amyloid-associated depression: a prodromal depression of Alzheimer disease? Arch Gen Psychiatry 2008;65:542-50.

18. Petersen RC, Smith GE, Waring SC, et al. Mild cognitive impairment: clinical characterization and outcome. Arch Neurol 1999;56:303-08.

19. Petersen RC, Morris JC. Mild cognitive impairment as a clinical entity and treatment target. Arch Neurol 2005;62:1160-63.

20. Caracciolo B, Palmer K, Monastero R, et al. Occurrence of cognitive impairment and dementia in the community: a 9-year-long prospective study. Neurology 2008;70:1778-85.

21. Larrieu S, Letenneur L, Orgogozo JM, et al. Incidence and outcome of mild cognitive impairment in a population-based prospective cohort. Neurology 2002;59:1594-99.

22. Fratiglioni L, Viitanen M, Backman L, et al. Occurrence of dementia in advanced age: the study design of the Kungsholmen Project. Neuroepidemiology 1992;11 Suppl 1:29-36.

23. WHO. International Classification of Disease, Injuries, and Cause of Death, 8th revision (ICD-8). Geneva: World Health Organization, 1967.

24. WHO. International Classification of Disease, Injuries, and Cause of Death, 9th revision (ICD-9). Geneva: World Health Organization, 1987.

25. Asberg M, Schalling D. Construction of a new psychiatric rating instrument, the Comprehensive Psychopathological Rating Scale (CPRS). Prog Neuropsychopharmacol 1979;3:405-12.

26. Berger AK, Fratiglioni L, Forsell Y, et al. The occurrence of depressive symptoms in the preclinical phase of AD: a population-based study. Neurology 1999;53:1998-2002.

27. Folstein MF, Folstein SE, McHugh PR. "Mini-mental state". A practical method for grading the cognitive state of patients for the clinician. J Psychiatr Res 1975;12:18998. 
28. Katz S. Assessing self-maintenance: activities of daily living, mobility, and instrumental activities of daily living. J Am Geriatr Soc 1983;31:721-27.

29. Wahlin A, Backman L, Winblad B. Free recall and recognition of slowly and rapidly presented words in very old age: a community-based study. Exp Aging Res 1995;21:251-71.

30. APA. Diagnostic and Statistical Manual of Mental DIsorders, Revised Third Edition (DSM-III-R). Washington, DC: American Psychiatric Association, 1987.

31. Fratiglioni L, Viitanen M, von Strauss E, et al. Very old women at highest risk of dementia and Alzheimer's disease: incidence data from the Kungsholmen Project, Stockholm. Neurology 1997;48:132-8.

32. Xu WL, Qiu CX, Wahlin A, et al. Diabetes mellitus and risk of dementia in the Kungsholmen project: a 6-year follow-up study. Neurology 2004;63:1181-6.

33. Monastero R, Palmer K, Qiu C, et al. Heterogeneity in risk factors for cognitive impairment, no dementia: population-based longitudinal study from the Kungsholmen Project. Am J Geriatr Psychiatry 2007;15:60-9.

34. Basun H, Corder EH, Guo Z, et al. Apolipoprotein E polymorphism and stroke in a population sample aged 75 years or more. Stroke 1996;27:1310-15.

35. Fratiglioni L, Wang HX, Ericsson $\mathrm{K}$, et al. Influence of social network on occurrence of dementia: a community-based longitudinal study. Lancet 2000;355:1315-19.

36. Padyukov L, Silva C, Stolt $\mathrm{P}$, et al. A gene-environment interaction between smoking and shared epitope genes in HLA-DR provides a high risk of seropositive rheumatoid arthritis. Arthritis Rheum 2004;50:3085-92.

37. Rubin DB. Multiple Imputation for Nonresponse in Surveys. New York: Wiley, 1987.

38. Kim J, Basak JM, Holtzman DM. The role of apolipoprotein E in Alzheimer's disease. Neuron 2009;63:287-303.

39. Bhalla RK, Butters MA, Becker JT, et al. Patterns of mild cognitive impairment after treatment of depression in the elderly. Am J Geriatr Psychiatry 2009;17:308-16.

40. Modrego PJ. Depression in the prodromal phase of Alzheimer disease and the reverse causal hypothesis. Arch Gen Psychiatry 2009;66:107.

41. Videbech P, Ravnkilde B. Hippocampal volume and depression: a meta-analysis of MRI studies. Am J Psychiatry 2004;161:1957-66. 


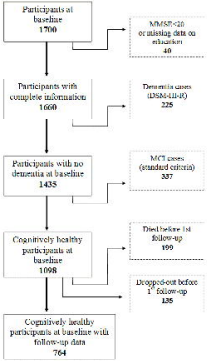


16

14

12

10

8

6

4

2

0
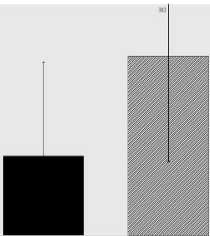

APOE-64/No low mood

Low mood/No APOE-s4

APOE-64 \& low mood 